\title{
Capacity and willingness of patients with chronic noncommunicable diseases to use information technology to help manage their condition: a cross-sectional study
}

\author{
Arash Ehteshami Afshar MD, Robert G. Weaver MSc, Meng Lin MSc, Michael Allan MD, \\ Paul E Ronksley PhD, Claudia Sanmartin PhD, Richard Lewanczuk MD, Mark Rosenberg PhD, \\ Braden Manns MD MSc, Brenda Hemmelgarn MD PhD, Marcello Tonelli MD SM, on behalf \\ of the Interdisciplinary Chronic Disease Collaboration*
}

\section{Abstract}

Background: Health care providers have shown considerable interest in using information technologies such as email, text messages and video conferencing to facilitate the management of chronic noncommunicable diseases such as hypertension, diabetes mellitus and vascular disease. We sought to determine whether these technologies are available and appealing to the target population.

Methods: We analyzed cross-sectional data from a computer-assisted telephone survey, conducted by Statistics Canada in February and March 2012, of western Canadian adults with at least 1 chronic condition. Survey respondents were asked about their capacity (e.g., "Do you own a mobile phone?") and willingness to use each of 3 information technologies (email, text messages and video conferencing) to interact with health care providers. For all analyses, Statistics Canada's calibrated design weights and bootstrap weights were used to obtain population-level point estimates for proportions and odds ratios.

Results: In total, 1849 (79.8\%) of 2316 eligible people participated. Of the 1849 participants, $81.9 \%$ had hypertension, $26.2 \%$ had diabetes, $21.4 \%$ had heart disease, and $7.9 \%$ had stroke; $32.2 \%$ had more than 1 of the 4 chronic conditions of interest. High proportions of respondents owned a computer with Internet access $(76.4 \%, 95 \%$ confidence interval $[\mathrm{Cl}] 73.3 \%-79.3 \%)$ or a mobile phone $(73.9 \%, 95 \% \mathrm{Cl} 70.7 \%-76.8 \%)$. About two-thirds of respondents were interested in using email to interact with a specialist $(66.3 \%$, $95 \% \mathrm{Cl} 63.0 \%-69.5 \%)$; respondents were less enthusiastic about using text messages (44.9\%, 95\% $\mathrm{Cl} 41.2 \%-48.7 \%)$. Enthusiasm for video conferencing was more pronounced among those residing further from medical specialists than among those living closer. Among respondents who were potentially interested in video conferencing, almost $50 \%$ of remote dwellers would use this technology if it saved more than 60 minutes of travel time.

Interpretation: Many people were interested in using electronic technologies, especially video conferencing and email-based methods, to help manage their chronic condition. The effectiveness and cost implications of using email and video conferencing in the management of chronic disease deserve further consideration.

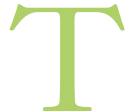
he management of chronic noncommunicable diseases such as hypertension, diabetes mellitus and vascular disease is a major challenge facing health services worldwide. ${ }^{1}$ Although most patients with chronic disease can be managed in primary care, many require referral to specialists, who generally practise in major urban centres. Given Canada's large size and low population density, an additional barrier to optimal management for some patients is that posed by the often considerable distances to specialists. ${ }^{2,3}$

Health care providers have shown considerable interest in using electronic technologies such as email, text messages (e.g., short message service, also known as "SMS") and video conferencing to facilitate management of chronic disease. ${ }^{4-6}$ Email could be used to send messages from provider to patient or vice versa and in theory might improve patients' access to providers or reduce providers' response time (especially for nonurgent issues). Text messages are not more than 160 characters in length and are usually received by mobile phones. Because of

Competing interests: None declared.

This article has been peer reviewed.

${ }^{*}$ The full membership list of the Interdisciplinary Chronic Disease Collaboration can be found at www.icdc.ca

Correspondence to: Marcello Tonelli, celloadm @ualberta.ca CMAJ Open 2014.DOI:10.9778/cmajo.20130070 
their brevity, they are best suited for reminders to the patient (e.g., to take medication) or motivational messages (e.g., "Did you go for a walk today?"), but they could also be used for brief queries from patient to provider. Video conferencing could replace face-to-face encounters with real-time, private interactions between the provider and the patient using specialized professional equipment or consumer-grade components (e.g., Skype running on a personal computer).

These technologies offer a potential solution to the geographic barriers faced by many Canadians with chronic disease and could be used to promote self-management regardless of where patients live. ${ }^{7}$ However, their utility depends on their availability and appeal to the target population. In particular, many Canadians with chronic disease are older and of lower socioeconomic status, ${ }^{8}$ which might reduce their capacity and willingness to use information technologies. ${ }^{9,10} \mathrm{We}$ assessed survey data from a large sample of western Canadians with chronic conditions to examine their capacity and willingness to use information technologies to facilitate the delivery of health care services, focusing on hypothetical interactions with a relevant medical specialist.

\section{Methods}

\section{Data sources}

The Canadian Community Health Survey (CCHS) is a biennial national survey that assesses the health status and potential determinants of health among Canadian residents aged 12 years and older. People residing on Aboriginal reserves, those living in long-term care facilities and members of the Canadian Forces are not eligible to participate in the CCHS. Further information on the CCHS (including response rates and measures of data quality) is available elsewhere. ${ }^{11,12}$ From February 1 to March 31, 2012, Statistics Canada used computerassisted telephone interviews to conduct a special survey that was designed by our group, the Interdisciplinary Chronic Disease Collaboration (www.icdc.ca). This special survey, the Barriers to Care for People with Chronic Health Conditions survey, was designed to elicit the perspectives of respondents about the current quality of their chronic disease care, including potential barriers and facilitators.

The Barriers to Care for People with Chronic Health Conditions survey gathered data from adults aged 40 years or older residing in the 4 western Canadian provinces (British Columbia, Alberta, Saskatchewan and Manitoba) who responded to the 2011 CCHS and who reported that they had received a diagnosis of diabetes, heart disease, hypertension or prior stroke. With participants' permission, responses were linked to their 2011 CCHS responses, which provided additional demographic and lifestyle information. The current study presents results from a priori analyses related to the use of information technologies.

\section{Capacity and willingness to use information technologies}

Survey respondents were asked about their capacity (e.g., "Do you own a mobile phone?") and willingness to use each of 3 information technologies (email, text messages, video conferencing) to interact with health care providers. Those who had the capacity but were unwilling were asked open-ended questions to explore the reasons for their unwillingness. In addition, a hypothetical scenario was used in asking participants how many minutes would have to be saved through a video conference encounter before they would be willing to use such an encounter to replace a face-to-face physician visit.

\section{Proximity to specialist care}

We classified residence location according to whether the respondent resided in the same city (census metropolitan area) as the closest relevant medical specialist's practice (e.g., cardiologist, endocrinologist, nephrologist, general internist), using postal codes to define the respondents' residence locations. Respondents with a relevant medical specialist in the same metropolitan area were considered to reside "in close proximity" to such specialists; other participants were considered to reside "further" from specialists.

\section{Other variables}

We categorized respondents by their chronic condition(s) and whether they reported diagnosis of 1 or more than 1 of the 4 chronic conditions. We obtained sociodemographic and health characteristics from the CCHS. We calculated body mass index from self-reported weight and height, using an adjustment for self-reported data. ${ }^{13}$

\section{Analysis}

We performed all analyses using STATA 11.0. The baseline characteristics of the survey respondents were compared across residence locations. The proportions of respondents reporting on key variables of interest were compared using design-based F tests. Respondents' stated reasons regarding why they were or were not interested in using the various technologies were also explored and presented graphically.

We used logistic regression modelling to examine the association between sociodemographic factors and the outcomes of interest (capacity and willingness to use the technologies). We adjusted the models for characteristics as described in the behavioural model of health service utilization ${ }^{14}$ and the motivational model ${ }^{15}$ which are frameworks for factors that may be associated with interest in electronic technologies. These variables were categorized as baseline characteristics, current quality of health care and attitude toward new technologies. In addition, we investigated respondents' attitudes regarding how much time would have to be saved to persuade them to use video conferencing for a specialist visit (i.e., < 30 minutes, 31-60 minutes, > 60 minutes or "don't know") rather than an in-person visit.

For all analyses, we applied Statistics Canada's calibrated design weights and bootstrap weights to obtain populationlevel point estimates for proportions and odds ratios and used bootstrapping to determine $95 \%$ confidence intervals (CIs) for the estimates. These weighting methods account for the complex sampling frame used to generate the CCHS. In accordance with Statistics Canada guidelines, if the coefficient of variation was $16 \%$ to $33.3 \%$, the results were considered 
potentially unreliable and were interpreted with caution. If the coefficient of variation was above $33.3 \%$, the results were considered unreliable and were not presented.

The Conjoint Health Research Ethics Board of the University of Calgary and the Health Research Ethics Board of the University of Alberta gave ethics approval for the study.

\section{Results}

Of the 2316 people selected, 1849 (79.8\%) completed the Barriers to Care for People with Chronic Health Conditions survey. The majority of these 1849 respondents were white (1609 [87.0\%]) and were married or in common-law relationships (1239 [67.0\%]) (Table 1). A substantial proportion of respondents were obese (body mass index 30 or above;
$30.8 \%$ ), and $69.2 \%$ were current or former smokers. The greatest number of respondents were from British Columbia (44.5\%), followed by Alberta (31.7\%), Manitoba (13.1\%) and Saskatchewan (10.8\%). Most respondents had received at least some postsecondary education or were university graduates (61.1\%), and almost half were $40-64$ years of age (48.8\%).

Overall, $1514(81.9 \%)$ of respondents had hypertension, $486(26.3 \%)$ had diabetes, $396(21.4 \%)$ had heart disease, and $147(8.0 \%)$ had stroke; $596(32.2 \%)$ had more than 1 of the 4 chronic conditions of interest. Despite having at least 1 chronic disease, $77.1 \%$ of respondents reported their health as "good" or better. Other health-related characteristics of the respondents are shown in Table 1 . Less than $1 \%$ of respondents reported having used one of the information technologies of interest to access health care in the past 12 months.

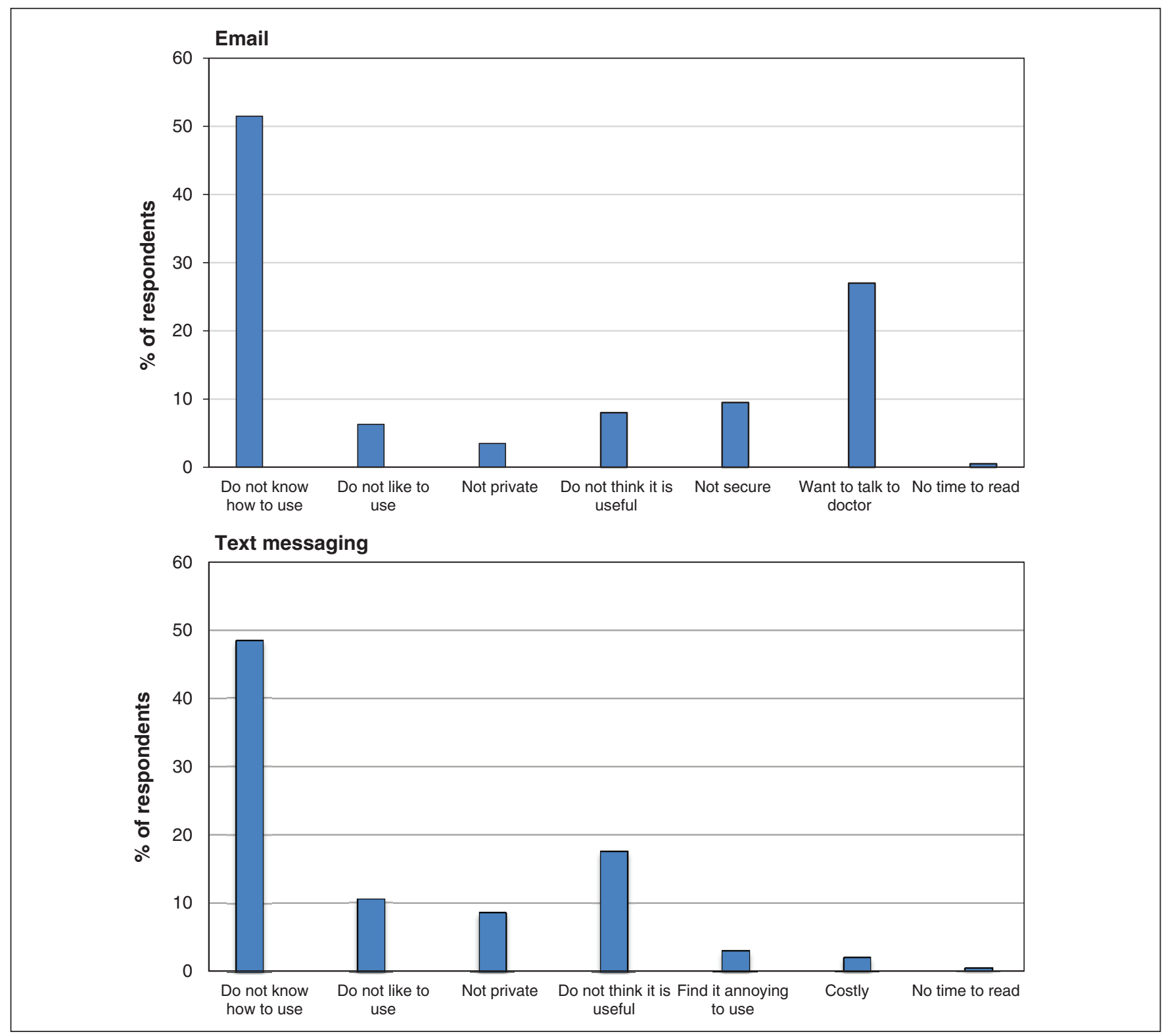

Figure 1: Reasons provided for lack of willingness to use email and text messages. For each mode of communication, the values do not sum to $100 \%$ because some respondents gave more than one reason, whereas others provided no reasons. 


\section{OPEN}

\begin{tabular}{|c|c|c|c|c|}
\hline \multirow[b]{2}{*}{ Characteristic } & \multicolumn{3}{|c|}{ Group, by proximity of residence to specialist; \% of respondents $(95 \% \mathrm{Cl})^{*}$} & \multirow[b]{2}{*}{$p$ value } \\
\hline & Total & $\begin{array}{l}\text { Close proximity to } \\
\text { specialist } †\end{array}$ & $\begin{array}{l}\text { Further from } \\
\text { specialist† }\end{array}$ & \\
\hline Sex, male & $49.9(46.0-53.8)$ & $50.6(44.2-57.0)$ & $49.2(45.0-53.4)$ & 0.7 \\
\hline \multicolumn{4}{|l|}{ Age category, yr } & \multirow[t]{4}{*}{0.04} \\
\hline $40-64$ & $48.8(45.6-52.0)$ & $50.8(45.0-56.5)$ & $46.8(42.5-51.1)$ & \\
\hline $65-74$ & $26.9(24.1-30.0)$ & $22.8(18.4-27.9)$ & $31.1(27.3-35.1)$ & \\
\hline$\geq 75$ & $24.3(21.7-27.1)$ & $26.4(21.7-31.8)$ & $22.2(19.3-25.3)$ & \\
\hline \multicolumn{4}{|l|}{ Level of education } & \multirow[t]{5}{*}{$<0.001$} \\
\hline $\begin{array}{l}\text { Did not graduate from } \\
\text { high school }\end{array}$ & $21.7(19.0-24.5)$ & $15.6(11.9-20.2)$ & $27.7(24.3-31.4)$ & \\
\hline High school graduation & $17.3(14.6-20.4)$ & $16.9(12.7-22.1)$ & $17.7(14.5-21.5)$ & \\
\hline $\begin{array}{l}\text { Some postsecondary } \\
\text { education (did not graduate) }\end{array}$ & $43.2(39.4-47.0)$ & $42.5(36.5-48.8)$ & $43.8(39.6-48.2)$ & \\
\hline University graduation & $17.9(14.7-21.5)$ & $25.0(19.3-31.7)$ & $10.7(8.4-13.7)$ & \\
\hline \multicolumn{4}{|l|}{ Household income, \$ } & \multirow[t]{5}{*}{0.001} \\
\hline$<25000$ & $15.2(12.9-17.8)$ & $13.0(9.4-17.7) \ddagger$ & $17.5(14.6-20.7)$ & \\
\hline $25000-39999$ & $18.5(15.9-21.3)$ & $16.3(12.6-20.9)$ & $20.6(17.6-23.9)$ & \\
\hline $40000-70000$ & $28.6(25.5-31.9)$ & $25.4(20.6-31.0)$ & $31.7(27.7-36.0)$ & \\
\hline$>70000$ & $37.8(33.9-41.8)$ & $45.3(38.7-52.0)$ & $30.2(26.3-34.5)$ & \\
\hline Has prescription drug insurance & $85.9(82.8-88.6)$ & $85.8(80.0-90.0)$ & $86.1(82.9-88.8)$ & 0.9 \\
\hline Married/common-law & $67.0(63.2-70.6)$ & $68.1(61.8-73.8)$ & $65.8(62.0-69.5)$ & 0.5 \\
\hline Country of birth Canada & $76.2(72.3-79.7)$ & $69.1(62.2-75.2)$ & $83.4(78.9-87.0)$ & $<0.001$ \\
\hline \multicolumn{4}{|l|}{ Province of residence } & \multirow[t]{5}{*}{$<0.001$} \\
\hline British Columbia & $44.5(41.4-47.7)$ & $39.6(34.0-45.6)$ & $49.4(45.3-53.5)$ & \\
\hline Alberta & $31.7(28.8-34.6)$ & $35.4(30.1-41.1)$ & $27.9(24.4-31.6)$ & \\
\hline Saskatchewan & $10.8(9.5-12.2)$ & $8.1(6.2-10.6)$ & $13.5(11.2-16.2)$ & \\
\hline Manitoba & $13.1(11.2-15.2)$ & $16.9(13.4-21.0)$ & $9.2(7.2-11.8)$ & \\
\hline Ethnic origin, white & $87.0(83.4-89.9)$ & $82.8(76.3-87.8)$ & $91.2(88.2-93.5)$ & 0.004 \\
\hline \multicolumn{5}{|l|}{ Chronic condition of interest } \\
\hline Hypertension & $81.9(78.9-84.5)$ & $80.7(75.4-85.1)$ & $83.0(79.4-86.1)$ & 0.4 \\
\hline Diabetes mellitus & $26.2(23.7-28.9)$ & $24.5(20.1-29.5)$ & $28.0(24.4-31.9)$ & 0.3 \\
\hline Heart disease & $21.4(18.7-24.3)$ & $19.7(15.5-24.7)$ & $23.1(19.5-27.1)$ & 0.3 \\
\hline Stroke & $7.9(6.4-9.6)$ & $7.0(4.7-10.2) \ddagger$ & $8.8(6.6-11.6)$ & 0.4 \\
\hline Additional chronic disease & $63.0(59.3-66.6)$ & $62.1(55.5-68.4)$ & $63.9(59.5-68.1)$ & 0.7 \\
\hline $\begin{array}{l}\text { Self-perceived health } \\
\text { excellent/very good/good }\end{array}$ & $77.1(74.1-84.5)$ & $80.3(75.2-84.5)$ & $73.9(69.9-77.5)$ & 0.05 \\
\hline \multicolumn{4}{|l|}{ Smoking status } & \multirow[t]{4}{*}{$<0.001$} \\
\hline Never & $30.8(27.0-34.9)$ & $38.3(31.8-45.2)$ & $23.2(19.9-26.9)$ & \\
\hline Occasional/former & $53.0(48.8-57.2)$ & $46.8(40.3-53.3)$ & $59.4(54.9-63.7)$ & \\
\hline Daily & $16.2(13.2-19.7)$ & $15.0(10.4-21.1) \ddagger$ & $17.4(14.1-21.3)$ & \\
\hline \multicolumn{4}{|l|}{ Alcohol use } & \multirow[t]{4}{*}{0.3} \\
\hline None & $26.0(22.6-29.6)$ & $26.9(21.7-32.9)$ & $25.0(21.4-29.0)$ & \\
\hline Occasional & $19.8(16.8-23.2)$ & $17.4(13.1-22.7)$ & $22.2(18.4-26.7)$ & \\
\hline Regular & $54.2(50.2-58.2)$ & $55.7(49.2-62.0)$ & $52.8(48.4-57.1)$ & \\
\hline Body mass index $<30$ & $69.2(65.3-72.9)$ & $70.7(64.1-76.6)$ & $67.7(63.6-71.6)$ & 0.4 \\
\hline \multicolumn{5}{|c|}{$\begin{array}{l}\text { Note: } \mathrm{Cl}=\text { confidence interval. } \\
\text { *All proportions and } 95 \% \text { Cls are weighted and bootstrapped as per Statistics Canada guidelines. } \\
\text { †Respondents with a relevant medical specialist in the same census metropolitan area as their home were considered to live "in close proximity" to } \\
\text { such specialists; other participants were considered to live "further" from specialists. } \\
\text { †Results must be interpreted with caution, as they may be unreliable (coefficient of variation falls between } 16 \% \text { and } 33.3 \% \text { ). }\end{array}$} \\
\hline
\end{tabular}


The majority of respondents owned a computer with Internet access $(76.4 \%, 95 \%$ CI $73.3 \%-79.3 \%)$ or a mobile phone $(73.9 \%$, 95\% CI 70.7\%-76.8\%). Nearly two-thirds were interested in using email to interact with a specialist (66.3\%, 95\% CI 63.0\%-69.5\%), but respondents were less enthusiastic about text messaging (44.9\%, 95\% CI $41.2 \%-$ $48.7 \%$ ) (Table 2). There was considerable interest in using video conferencing to interact with physicians, but this interest was less pronounced for primary care $(50.4 \%, 95 \%$ CI $46.4 \%-54.4 \%)$ than for specialized care $(65.1 \%, 95 \%$ CI $61.4 \%-68.6 \%)$, perhaps because respondents perceived fewer barriers to interacting with primary care physicians. As expected, enthusiasm for video conferencing was more pronounced among those residing further from (v. closer to) medical specialists.

The most common reasons given by respondents for unwillingness to correspond with a provider by email or text message are shown in Figure 1 and Appendix 1 (Appendix available at www.cmajopen.ca/content/2/2/E51/suppl/DC1).

On the basis of hypothetical scenarios, those residing further from specialists stated that they would need to save more time before they would be willing to visit a video conferencing centre instead of attending a face-to-face visit with a specialist (compared with those living in close proximity to a specialist; Table 2). Among the respondents who were potentially interested in video conferencing, almost $50 \%$ of those residing further from a specialist would use video conferencing if it could save more than 60 minutes of their time. However, nearly one-third of respondents who were potentially interested in video conferencing would use a videoconference visit in preference to a face-to-face visit in exchange for saving $30 \mathrm{~min}$ utes of their time or less, which indicates that even relatively small time savings may be potentially appealing to patients.

Multivariable logistic regression analyses identified the following characteristics as being independently associated with willingness to visit a video conferencing centre for specialist care (Table 3): living further from a specialist, having at least some postsecondary education and having a lower burden of chronic disease. Interest in using email to interact with a specialist was independently associated with age 40-64 years (v. older), white ethnic origin, having an Internet connection at home and having a university degree. Interest in using text

Table 2: Capacity and willingness to use information technology

Group, by proximity of residence to specialist; \% of respondents $(95 \% \mathrm{Cl})^{*}$

\begin{tabular}{|c|c|c|c|c|}
\hline \multirow[b]{2}{*}{ Variable } & & & & \multirow[b]{2}{*}{$p$ value } \\
\hline & Total & $\begin{array}{l}\text { Close proximity to } \\
\text { specialist } †\end{array}$ & $\begin{array}{l}\text { Further from } \\
\text { specialist } \dagger\end{array}$ & \\
\hline \multicolumn{5}{|l|}{ Availability of equipment } \\
\hline $\begin{array}{l}\text { Own computer with Internet } \\
\text { access }\end{array}$ & $76.4(73.3-79.3)$ & $80.2(74.9-84.6)$ & $72.6(68.8-76.1)$ & 0.02 \\
\hline Own a cell phone & $73.9(70.7-76.8)$ & $75.6(70.4-80.2)$ & $72.1(68.0-75.9)$ & 0.3 \\
\hline \multicolumn{5}{|l|}{$\begin{array}{l}\text { Willingness to use } \\
\text { technologies }\end{array}$} \\
\hline $\begin{array}{l}\text { Video conferencing to } \\
\text { interact with primary care } \\
\text { provider }\end{array}$ & $50.4(46.4-54.4)$ & $47.1(40.2-54.1)$ & $53.8(49.5-58.1)$ & 0.1 \\
\hline $\begin{array}{l}\text { Video conferencing to } \\
\text { interact with specialist }\end{array}$ & $65.1(61.4-68.6)$ & $60.1(53.7-66.2)$ & $70.2(66.4-73.7)$ & 0.006 \\
\hline $\begin{array}{l}\text { Email to interact with } \\
\text { specialist }\end{array}$ & $66.3(63.0-69.5)$ & $69.0(62.9-74.5)$ & $63.5(59.7-67.2)$ & 0.1 \\
\hline $\begin{array}{l}\text { Text messaging to interact } \\
\text { with specialist }\end{array}$ & $44.9(41.2-48.7)$ & $46.2(39.8-52.7)$ & $43.6(39.3-48.0)$ & 0.5 \\
\hline \multicolumn{5}{|l|}{$\begin{array}{l}\text { Threshold time saved for } \\
\text { use of video } \\
\text { conferencing, min } ¥\end{array}$} \\
\hline$\leq 30$ & $28.3(23.9-33.0)$ & $30.6(23.1-39.4)$ & $26.2(21.5-31.5)$ & \multirow[t]{4}{*}{0.04} \\
\hline $31-60$ & $21.4(17.2-26.4)$ & $25.7(18.2-35.0) \S$ & 17.7 (13.9-22.3) & \\
\hline$>60$ & $41.7(36.9-46.7)$ & $34.1(26.6-42.5)$ & $48.3(42.8-53.9)$ & \\
\hline Don't know & $8.6(6.5-11.4)$ & $9.6(5.9-15.1) \S$ & $7.8(5.5-10.9) \S$ & \\
\hline
\end{tabular}

Note: $\mathrm{Cl}=$ confidence interval.

${ }^{*}$ All proportions and $95 \%$ Cls are weighted and bootstrapped as per Statistics Canada guidelines.

†Respondents with a relevant medical specialist in the same census metropolitan area as their home were considered to reside "in close proximity" to such specialists; other participants were considered to reside "further" from specialists.

¥Minimum amount of travel time saved by use of video conferencing for a respondent to prefer this technology over an in-person visit with a specialist (among respondents who were willing to use video conferencing).

$\S R$ Rults must be interpreted with caution, as they may be unreliable (coefficient of variation falls between 16\% and 33.3\%). 
Table 3 (part 1 of 2): Factors associated with willingness to use information technology

\begin{tabular}{|c|c|c|c|c|c|c|}
\hline \multirow[b]{2}{*}{ Factor } & \multicolumn{2}{|c|}{ Email } & \multicolumn{2}{|c|}{ Text messaging } & \multicolumn{2}{|c|}{ Video conferencing } \\
\hline & $\begin{array}{l}\text { Interest, \% } \\
(95 \% \mathrm{Cl})\end{array}$ & $\begin{array}{l}\text { Adjusted OR } \\
(95 \% \mathrm{Cl})\end{array}$ & $\begin{array}{l}\text { Interest, \% } \\
(95 \% \mathrm{Cl})\end{array}$ & $\begin{array}{l}\text { Adjusted OR } \\
(95 \% \mathrm{Cl})\end{array}$ & $\begin{array}{l}\text { Interest, \% } \\
(95 \% \mathrm{Cl})\end{array}$ & $\begin{array}{l}\text { Adjusted OR } \\
(95 \% \mathrm{Cl})\end{array}$ \\
\hline \multicolumn{7}{|l|}{ Residence location } \\
\hline Close to specialist & $69.0(62.9-74.5)$ & Ref & $46.2(39.8-52.7)$ & Ref & $60.1(53.7-66.2)$ & Ref \\
\hline Further from specialist & $63.5(59.7-67.2)$ & $1.08(0.66-1.74)$ & $43.6(39.3-48.0)$ & $1.21(0.82-1.76)$ & $70.2(66.4-73.7)$ & $2.00(1.37-2.90)$ \\
\hline \multicolumn{7}{|l|}{ Have access to Internet } \\
\hline No & $16.4(12.6-21.2)$ & Ref & $23.0(18.3-28.5)$ & Ref & $52.0(44.8-59.1)$ & Ref \\
\hline Yes & $81.7(78.1-84.9)$ & $12.76(7.43-21.90)$ & $52.7(47.2-56.1)$ & $2.05(1.30-3.22)$ & $69.2(64.9-73.3)$ & $1.40(0.85-2.31)$ \\
\hline \multicolumn{7}{|l|}{ Own cell phone } \\
\hline No & $48.7(41.9-55.6)$ & Ref & $27.3(22.4-32.8)$ & Ref & $57.5(50.6-64.2)$ & Ref \\
\hline Yes & $72.3(68.5-75.9)$ & $0.92(0.57-1.49)$ & $52.6(46.9-56.2)$ & $1.60(1.08-2.37)$ & $68.4(64.1-72.4)$ & $1.10(0.76-1.62)$ \\
\hline \multicolumn{7}{|l|}{ Sex } \\
\hline Men & $69.6(64.5-74.4)$ & Ref & $49.1(43.5-54.9)$ & Ref & $66.1(60.7-71.2)$ & Ref \\
\hline Women & $63.0(58.1-67.6)$ & $0.92(0.56-1.50)$ & $40.6(35.5-46.0)$ & $0.87(0.60-1.25)$ & $64.0(58.8-68.9)$ & $1.00(0.68-1.47)$ \\
\hline \multicolumn{7}{|l|}{ Age, yr } \\
\hline $40-64$ & $80.1(75.2-84.2)$ & Ref & $55.8(49.6-61.8)$ & Ref & $70.3(64.6-75.4)$ & Ref \\
\hline $65-74$ & $64.4(57.8-70.4)$ & $0.75(0.44-1.28)$ & $41.2(34.6-48.1)$ & $0.81(0.53-1.23)$ & $64.5(57.7-70.8)$ & $0.83(0.54-1.28)$ \\
\hline$\geq 75$ & $40.7(34.3-47.5)$ & $0.46(0.25-0.81)$ & $26.9(21.8-32.5)$ & $0.61(0.38-0.99)$ & $55.3(48.5-61.9)$ & $0.81(0.50-1.32)$ \\
\hline \multicolumn{7}{|l|}{ Level of education } \\
\hline $\begin{array}{l}\text { Did not graduate from } \\
\text { high school }\end{array}$ & $38.0(31.7-44.7)$ & Ref & $28.6(23.1-34.8)$ & Ref & $49.8(43.3-56.3)$ & Ref \\
\hline High school graduation & $68.8(59.6-76.7)$ & $1.74(0.96-3.16)$ & $44.2(34.8-54.1)$ & $1.37(0.81-2.31)$ & $61.8(52.3-70.4)$ & $1.39(0.83-2.30)$ \\
\hline $\begin{array}{l}\text { Some postsecondary (did } \\
\text { not graduate) }\end{array}$ & $70.5(64.9-75.5)$ & $1.60(0.97-2.63)$ & $46.6(40.7-52.6)$ & $1.33(0.85-2.08)$ & $68.2(62.1-73.8)$ & $2.05(1.28-3.26)$ \\
\hline University graduation & $89.1(82.5-93.5)$ & $4.41(1.98-9.81)$ & $62.4(51.5-72.1)$ & $2.31(1.22-4.37)$ & $79.0(71.5-84.9)$ & $3.89(2.07-7.31)$ \\
\hline \multicolumn{7}{|l|}{ Household income, \$ } \\
\hline$<25000$ & $41.0(32.4-50.1)$ & Ref & $26.4(20.0-34.1)$ & Ref & $55.3(45.9-64.4)$ & Ref \\
\hline $25000-39999$ & $50.1(42.3-57.9)$ & $0.92(0.49-1.75)$ & $29.7(23.4-36.8)$ & $1.09(0.61-1.94)$ & $60.6(52.3-68.4)$ & $1.05(0.56-1.97)$ \\
\hline $40000-70000$ & $69.8(63.7-75.3)$ & $1.38(0.75-2.55)$ & $45.3(38.8-52.0)$ & $1.49(0.84-2.67)$ & $70.2(64.1-75.6)$ & $1.12(0.59-2.15)$ \\
\hline$>70000$ & $81.4(74.9-86.6)$ & $1.35(0.64-2.87)$ & $59.4(52.0-66.4)$ & $2.05(1.11-3.77)$ & $67.2(59.8-73.9)$ & $0.71(0.34-1.47)$ \\
\hline \multicolumn{7}{|l|}{$\begin{array}{l}\text { Prescription drug } \\
\text { insurance status }\end{array}$} \\
\hline No & $68.2(59.6-75.7)$ & Ref & $40.4(30.1-51.7)$ & Ref & $67.1(58.0-75.1)$ & Ref \\
\hline Yes & $66.0(62.2-69.5)$ & $0.72(0.42-1.22)$ & $45.7(41.6-49.8)$ & $1.15(0.70-1.87)$ & $64.8(60.7-68.6)$ & $0.79(0.48-1.28)$ \\
\hline \multicolumn{7}{|l|}{ Marital status } \\
\hline Married/common-law & $72.3(68.2-76.1)$ & Ref & $47.4(42.5-52.2)$ & Ref & $66.1(61.1-70.7)$ & Ref \\
\hline $\begin{array}{l}\text { Widowed/separated/ } \\
\text { divorced /single }\end{array}$ & $54.0(47.8-60.0)$ & $0.85(0.54-1.36)$ & $40.1(34.1-46.4)$ & $1.33(0.88-1.99)$ & $62.9(57.4-68.1)$ & $0.96(0.64-1.46)$ \\
\hline \multicolumn{7}{|l|}{ Country of birth } \\
\hline Canada & $67.1(63.6-70.5)$ & Ref & $47.9(43.5-52.3)$ & Ref & $66.5(62.6-70.2)$ & Ref \\
\hline Outside Canada & $63.4(53.9-72.0)$ & $1.32(0.74-2.36)$ & $35.3(26.8-44.9)$ & $0.80(0.47-1.35)$ & $60.4(50.4-69.7)$ & $0.84(0.51-1.38)$ \\
\hline \multicolumn{7}{|l|}{ Province of residence } \\
\hline Alberta & $70.4(64.6-75.5)$ & Ref & $47.7(40.8-54.7)$ & Ref & $69.1(62.0-75.4)$ & Ref \\
\hline British Columbia & $69.2(63.4-74.4)$ & $0.77(0.44-1.36)$ & $42.1(36.1-48.3)$ & $0.79(0.51-1.23)$ & $66.0(60.1-71.4)$ & $0.84(0.51-1.38)$ \\
\hline Saskatchewan & $62.7(54.4-70.4)$ & $0.87(0.50-1.52)$ & $44.0(35.7-52.7)$ & $0.87(0.53-1.43)$ & $59.4(50.9-67.4)$ & $0.62(0.36-1.08)$ \\
\hline Manitoba & $49.7(39.1-60.3)$ & $0.43(0.19-0.97)$ & $48.6(37.2-59.9)$ & $1.23(0.68-2.23)$ & $57.0(45.6-67.7)$ & $0.60(0.31-1.16)$ \\
\hline \multicolumn{7}{|l|}{ Ethnic origin } \\
\hline White & $68.6(65.3-71.8)$ & Ref & $45.9(41.9-50.0)$ & Ref & $65.3(61.5-68.9)$ & Ref \\
\hline Aboriginal/other & $50.5(36.3-64.6)$ & $0.32(0.13-0.76)$ & $38.1(25.6-52.4) \dagger$ & $0.71(0.36-1.40)$ & $63.7(50.6-75.0)$ & $1.28(0.68-2.43)$ \\
\hline
\end{tabular}


Table 3 (part 2 of 2): Factors associated with willingness to use information technology*

\begin{tabular}{|c|c|c|c|c|c|c|}
\hline \multirow[b]{2}{*}{ Factor } & \multicolumn{2}{|c|}{ Email } & \multicolumn{2}{|c|}{ Text messaging } & \multicolumn{2}{|c|}{ Video conferencing } \\
\hline & $\begin{array}{l}\text { Interest, \% } \\
(95 \% \mathrm{Cl})\end{array}$ & $\begin{array}{l}\text { Adjusted OR } \\
(95 \% \mathrm{Cl})\end{array}$ & $\begin{array}{l}\text { Interest, \% } \\
(95 \% \mathrm{Cl})\end{array}$ & $\begin{array}{l}\text { Adjusted OR } \\
(95 \% \mathrm{Cl})\end{array}$ & $\begin{array}{l}\text { Interest, \% } \\
(95 \% \mathrm{Cl})\end{array}$ & $\begin{array}{l}\text { Adjusted OR } \\
(95 \% \mathrm{Cl})\end{array}$ \\
\hline \multicolumn{7}{|l|}{$\begin{array}{l}\text { Chronic condition of } \\
\text { interestł }\end{array}$} \\
\hline \multicolumn{7}{|l|}{ Hypertension } \\
\hline No & $66.4(57.2-74.6)$ & Ref & $43.7(34.3-53.6)$ & Ref & $63.8(54.6-72.1)$ & Ref \\
\hline Yes & $66.3(62.6-69.8)$ & $0.75(0.42-1.31)$ & $45.2(41.0-49.4)$ & $1.14(0.69-1.89)$ & $65.4(61.5-69.1)$ & $0.87(0.52-1.45)$ \\
\hline \multicolumn{7}{|l|}{ Diabetes mellitus } \\
\hline No & $68.0(64.0-71.8)$ & Ref & $45.1(40.6-49.6)$ & Ref & $66.2(61.8-70.4)$ & Ref \\
\hline Yes & $61.4(54.8-67.7)$ & $0.84(0.47-1.49)$ & $44.5(37.5-51.7)$ & $1.20(0.82-1.77)$ & $61.9(55.3-68.0)$ & $0.83(0.55-1.24)$ \\
\hline \multicolumn{7}{|l|}{ Heart disease } \\
\hline No & $68.5(64.7-72.0)$ & Ref & $44.8(40.5-49.1)$ & Ref & $66.9(62.8-70.7)$ & Ref \\
\hline Yes & $58.3(50.8-65.5)$ & $0.82(0.47-1.43)$ & $45.5(38.0-53.1)$ & $1.41(0.94-2.10)$ & $58.3(49.9-66.2)$ & $0.73(0.46-1.17)$ \\
\hline \multicolumn{7}{|l|}{ Stroke } \\
\hline No & $68.2(64.8-71.4)$ & Ref & $46.4(42.4-50.4)$ & Ref & $66.7(62.9-70.3)$ & Ref \\
\hline Yes & $43.9(31.7-57.0)$ & $0.73(0.37-1.45)$ & $28.0(18.6-39.8) \dagger$ & $0.73(0.40-1.35)$ & $46.0(33.2-59.3)$ & $0.55(0.28-1.07)$ \\
\hline \multicolumn{7}{|l|}{$\begin{array}{l}\text { Additional chronic } \\
\text { disease }\end{array}$} \\
\hline No & $75.3(70.1-79.8)$ & Ref & $50.2(43.8-56.5)$ & Ref & $75.4(70.7-79.6)$ & Ref \\
\hline Yes & $61.0(56.7-65.2)$ & $0.74(0.46-1.17)$ & $41.8(36.8-47.0)$ & $0.92(0.62-1.36)$ & $59.0(54.0-63.8)$ & $0.56(0.38-0.83)$ \\
\hline \multicolumn{7}{|l|}{ Self-perceived health } \\
\hline Excellent/very good/good & $69.9(66.1-73.5)$ & Ref & $46.1(41.9-50.5)$ & Ref & $68.1(64.0-72.0)$ & Ref \\
\hline Fair/poor & $53.8(46.4-61.0)$ & $0.85(0.49-1.46)$ & $40.9(33.2-49.1)$ & $1.12(0.70-1.77)$ & $54.8(47.4-61.9)$ & $0.79(0.52-1.19)$ \\
\hline
\end{tabular}

messaging to interact with a specialist was significantly associated with younger age, owning a mobile phone, having an Internet connection at home, having a university degree and higher income (Table 3).

\section{Interpretation}

New information technologies, such as email, text messaging and video conferencing, have the potential to improve chronic disease care. Although there appears to be interest among health care providers in adopting these technologies, ${ }^{6,16,17}$ the extent of interest among patients is less well described, especially among older adults with chronic disease. In this large survey of community-dwelling western Canadians with chronic disease, we assessed patients' self-reported interest in adopting these 3 types of information technology, focusing on interactions with specialists.

Participants expressed substantial interest in using information technology for chronic disease care, which indicates that these technologies warrant further consideration for their potential to improve physician-patient interactions, perhaps at lower cost than with current models of care. Our survey focused on interactions involving specialist care, but it appears that video conference visits with primary care physicians may also be of interest to patients. Like others, we found that younger and more educated respondents were more interested in all 3 forms of information technology. ${ }^{18}$ However, most respondents in the current study expressed interest in and capacity to use email and video conferencing regardless of age and educational status. The high level of interest in these technologies suggests the need for further research into their potential benefits, especially given that less than $1 \%$ of respondents had used these technologies to access health services in the year before the survey. Conversely, given that the capacity for and interest in email and text messaging were substantially lower among people in the lowest income category (< \$25 000 annually), our findings suggest that alternative strategies will be needed for this population.

There was consistently greater interest in email and video conferencing than in text messaging, especially among older participants. The availability of all 3 technologies is relatively high, so it is possible that fewer respondents were interested in text messaging because it was less familiar to them than the other 2 technologies; alternatively, they may have felt constrained by the short length of text messages (maximum 160 characters). However, of the 3 technologies considered in the 


\section{OPEN}

current study, the least is known about potential barriers to the use of text messaging, ${ }^{19}$ so this suggestion is speculative.

Prior systematic reviews have suggested that various electronic interventions can improve process-based or clinical outcomes in diabetes, ${ }^{20}$ chronic heart failure, ${ }^{21}$ respiratory conditions, ${ }^{22,23}$ smoking cessation programs ${ }^{24}$ and secondary prevention of coronary artery disease. A Cochrane systematic review showed that text message support might improve the self-management of chronic conditions such as diabetes, hypertension and asthma. ${ }^{25}$ Another review found promising results for text messaging as an intervention tool for weight loss. ${ }^{26}$

Several surveys have shown that patients are willing to use information technologies ${ }^{27}$ for routine care $^{15}$ or specialized care such as mental health services, ${ }^{26,28}$ especially if no physician is available locally ${ }^{29}$ or if the technologies would save them time and money. ${ }^{30}$ In our survey, the major reported barrier to uptake of email or text messaging was lack of knowledge, but our respondents appeared more optimistic than those in previous surveys about the potential merits of an electronic encounter: less than $30 \%$ cited a preference for a face-to-face visit as a major barrier to using email to communicate with a physician. In our study, few participants cited concerns about cost as a major barrier to use of text messaging. Previous studies have suggested that many patients would be willing to pay a small annual fee for certain online services such as viewing parts of their medical record, messaging with their physician, medication refills, appointment requests and billing inquiries. ${ }^{31}$ Finally, although increasing the use of information technology in health care communication will require careful attention to ensure that personal data remain confidential, ${ }^{32}$ concerns about privacy were not often cited by participants in our study as a potential barrier.

\section{Limitations}

Our study had some limitations. First, although the response rate was relatively high, the sample was drawn from respondents to the CCHS survey who resided in 4 Canadian provinces; thus, the generalizability of our findings to the wider Canadian population is uncertain. Furthermore, people living on Aboriginal reserves, those in long-term care and members of the Canadian Forces were not included in our analysis. Second, the data were self-reported, and relationships with actual behaviour were not examined. Third, we focused on certain important chronic conditions (hypertension, diabetes, heart disease and stroke) and the number of respondents with more than 1 of these conditions was small. Fourth, our index of residence location was unsophisticated, largely because privacy considerations precluded us from assessing the precise residence location of each respondent. However, given the relatively high level of interest in electronic technologies among both urban and nonurban respondents, this factor is unlikely to have affected our conclusions. Fifth, we considered 3 different types of information technology (each with its own strengths and limitations) in this analysis, whereas it might have been preferable to focus on 1 type and to examine specific characteristics that made its use more or less appealing. Sixth, although most patients with hyperten- sion, diabetes or heart disease can be managed in primary care, we considered electronic technologies largely as a means of facilitating specialist care. Future studies should further address how electronic technologies can be used to enhance management of chronic diseases in primary care. Finally, because of the study design, the questions in the survey were quite general. It would have been preferable to provide specific examples of how the information technologies would work, ideally with reference to a working prototype. For example, specifying that video conferencing could be done at home using Skype software and a webcam might have been associated with greater enthusiasm among respondents, as compared with video conferencing at a nearby health facility. This limitation should be addressed in future work.

\section{Conclusion}

The majority of western Canadians who responded to our survey were interested in using electronic technologies, especially video conferencing and email-based methods, to help manage their chronic disease. Although younger patients were more likely to be interested in and able to use such technologies, there was substantial interest among those aged 75 years and older. These findings suggest that email and video conferencing should be further explored as potential mechanisms for helping Canadians manage chronic diseases such as hypertension, diabetes and vascular disease.

\section{References}

1. Murray CJ, Vos T, Lozano R, et al. Disability-adjusted life years (DALYs) for 291 diseases and injuries in 21 regions, 1990-2010: a systematic analysis for the Global Burden of Disease Study 2010 [published erratum in Lancet 2013; 23:381:628]. Lancet 2012;380:2197-223.

2. Rucker D, Hemmelgarn BR, Lin M, et al. Quality of care and mortality are worse in chronic kidney disease patients living in remote areas. Kidney Int 2011;79:210-7.

3. Tonelli M, Hemmelgarn B, Kim AK, et al. Association between residence location and likelihood of kidney transplantation in Aboriginal patients treated with dialysis in Canada. Kidney Int 2006;70:924-30.

4. Sittig DF, King S, Hazlehurst BL. A survey of patient-provider e-mail communication: What do patients think? Int 7 Med Inform 2001;61:71-80.

5. Virji A, Yarnall KS, Krause KM, et al. Use of email in a family practice setting: opportunities and challenges in patient- and physician-initiated communication. BMC Med 2006; 4:18.

6. Katz SJ, Nissan N, Moyer CA. Crossing the digital divide: evaluating online communication between patients and their providers. Am 7 Manag Care 2004;10:593-8

7. Canada Health Infoway. Telehealth benefits and adoption: connecting people and providers across Canada. Stamford (CT): Gartner; 2011. Available: www.infoway-info route.ca/index.php/resources/reports/doc_download/447-telehealth-benefits-and -adoption-connecting-people-and-providers-full (accessed 2014 Apr. 8).

8. Chronic disease risk factors. Ottawa (ON): Public Health Agency of Canada; 2013 Available: www.phac-aspc.gc.ca/cd-mc/risk_factors-facteurs_risque-eng.php (accessed 2013 Sep. 5).

9. Table 358-0153 - Canadian Internet use survey, Internet use, by age group, Internet activity, sex, level of education and household income. Ottawa (ON): Statistics Canada; 2013. Available: www5.statcan.gc.ca/cansim/a26?lang=eng\&retrLang=eng\&id $=3580153 \&$ pattern $=$ internet $\&$ tabMode $=$ data Table $\& \operatorname{srchL}$ an $=-1 \& p 1=1 \& \mathrm{p} 2=50$ (accessed 2014 Apr. 8).

10. Table 358-0167 - Canadian Internet use survey, housebold access to the Internet at bome, by household income quartile, Canada and provinces. Ottawa (ON): Statistics Canada; 2013. Available: www5.statcan.gc.ca/cansim/a26?lang=eng\&retrLang =eng\&id=3580167\&pattern=internet $\&$ tabMode=data Table \&srchLan=-1\&p1=1 \&p2=50 (accessed 2013 Sep. 5).

11. Canadian community health survey - annual component (CCHS). Ottawa (ON): Statistics Canada; 2013. Available: www23.statcan.gc.ca/imdb/p2SV.pl?Function =getSurvey\&SDDS=3226\&Item_Id=144171\&lang=en (accessed 2013 Dec. 9).

12. Canadian community bealth survey (CCHS) - annual component. User guide 2010 and 2009-2010 microdata files. Ottawa (ON): Statistics Canada; 2011. Available: www23.statcan.gc.ca/imdb-bmdi/pub/document/3226_D7_T9_V8-eng.htm (accessed 2013 Dec. 9). 
13. Shields M, Connor Gorber S, Janssen I, et al. Bias in self-reported estimates of obesity in Canadian health surveys: an update on correction equations for adults. Health Rep 2011;22:35-45.

14. Aday LA, Andersen R. A framework for the study of access to medical care. Health Serv Res 1974;9:208-20.

15. Werner P, Karnieli E. A model of the willingness to use telemedicine for routine and specialized care. 7 Telemed Telecare 2003;9:264-72.

16. Larcher B, Arisi E, Berloffa F, et al. Analysis of user-satisfaction with the use of a teleconsultation system in oncology. Med Inform Internet Med 2003;28:73-84.

17. Cloutier P, Cappelli M, Glennie JE, et al. Mental health services for children and youth: a survey of physicians' knowledge, attitudes and use of telehealth services. 7 Telemed Telecare 2008;14:98-101.

18. Zickuhr K, Madden M. Older adults and internet use. Washington (DC): Pew Research Centre; 2012.

19. Jimison H, Gorman P, Woods S, et al. Barriers and drivers of health information technology use for the elderly, chronically ill, and underserved. Evid Rep Technol Assess (Full Rep) 2008;(175):1-1422.

20. Neubeck L, Redfern J, Fernandez R, et al. Telehealth interventions for the secondary prevention of coronary heart disease: a systematic review. Eur 7 Cardiovasc Prev Rehabil 2009;16:281-9.

21. Clark RA, Inglis SC, McAlister FA, et al. Telemonitoring or structured telephone support programmes for patients with chronic heart failure: systematic review and meta-analysis. BM7 2007;334:942.

22. Jaana $M$, Pare G, Sicotte C. Home telemonitoring for respiratory conditions: a systematic review. Am 7 Manag Care 2009;15:313-20.

23. Polisena J, Tran K, Cimon K, et al. Home telehealth for chronic obstructive pulmonary disease: a systematic review and meta-analysis. 7 Telemed Telecare 2010;16:120-7.

24. Myung SK, McDonnell DD, Kazinets G, et al. Effects of Web- and computerbased smoking cessation programs: meta-analysis of randomized controlled trials. Arch Intern Med 2009;169:929-37.

25. de Jongh T, Gurol-Urganci I, Vodopivec-Jamsek V, et al. Mobile phone messaging for facilitating self-management of long-term illnesses. Cochrane Database Syst Rev 2012;(12):CD007459.

26. Shaw R, Bosworth H. Short message service (SMS) text messaging as an intervention medium for weight loss: a literature review. Health Informatics 7. 2012; 18:235-50.

27. Kelly JM, Schwamm LH, Bianchi MT. Sleep telemedicine: a survey study of patient preferences. ISRN Neurol 2012;2012:135329.

28. Rohland BM, Saleh SS, Rohrer JE, et al. Acceptability of telepsychiatry to a rural population. Psychiatr Serv 2000;51:672-4.
29. Brick JE, Bashshur RL, Brick JF, et al. Public knowledge, perception, and expressed choice of telemedicine in rural West Virginia. Telemed f 1997;3:159-71.

30. Eikelboom RH, Atlas MD. Attitude to telemedicine, and willingness to use it, in audiology patients. F Telemed Telecare 2005;11 Suppl 2:S22-5.

31. Adler KG. Web portals in primary care: an evaluation of patient readiness and willingness to pay for online services. 7 Med Internet Res 2006;8:e26.

32. Avancha S, Baxi A, Kotz D. Privacy in mobile technology for personal healthcare. ACM Comput Surv 2012;45:1-54.

Affiliations: Department of Medicine (Afshar, Allan, Lin, Tonelli), University of Alberta, Edmonton, Alta.; Department of Medicine (Weaver, Hemmelgarn), University of Calgary, Calgary, Alta.; Department of Family Medicine (Allan), University of Alberta, Edmonton, Alta.; Department of Community Health Sciences (Ronksley, Manns), Faculty of Medicine, University of Calgary, Calgary, Alta.; Health Analysis Division (Sanmartin), Statistics Canada, Ottawa, Ont.; Division of Endocrinology and Metabolism (Lewanczuk), University of Alberta, Edmonton, Alta.; Department of Geography (Rosenberg), Queen's University, Kingston, Ont.

Contributors: Arash Ehteshami Afshar, Meng Lin, Braden Manns, Brenda Hemmelgarn and Marcello Tonelli contributed to study conception and design and to the acquisition, analysis or interpretation of data. Robert Weaver, Michael Allan, Paul E Ronksley, Claudia Sanmartin, Richard Lewanczuk and Mark Rosenberg also contributed to the interpretation of the data. All authors contributed to drafting the article or revising it critically for important intellectual content and provided final approval of the version to be published. Marcello Tonelli is the study guarantor.

Funding: This work was funded by an interdisciplinary team grant to the Interdisciplinary Chronic Disease Collaboration from Alberta InnovatesHealth Solutions. The views expressed in this paper are solely those of the authors and do not reflect those of Statistics Canada.

Supplemental information: For reviewer comments and the original submission of this manuscript, please see www.cmajopen.ca/content/2/2 /E51/suppl/DC1 